\title{
Childhood Cerebellar Anaplastic Astrocytoma
}

National Cancer Institute

\section{Source}

National Cancer Institute. Childhood Cerebellar Anaplastic Astrocytoma. NCI Thesaurus.

Code C115201.

A cerebellar anaplastic astrocytoma that occurs during childhood. 